\title{
Numerical and experimental investigation on the breast cancer tumour parameters by inverse heat transfer method using genetic algorithm and image processing
}

\author{
MARYAM BAHADOR ${ }^{1}$, MOHAMMAD MEHDI KESHTKAR ${ }^{2, *}$ and AMIN ZARIEE ${ }^{2}$ \\ ${ }^{1}$ Radiotherapy and Oncology Department, Shafa Hospital, Kerman University of Medical Sciences, Kerman, \\ Iran \\ ${ }^{2}$ Department of Mechanical Engineering, Kerman Branch, Islamic Azad University, Kerman, Iran \\ e-mail: mkeshtkar54@yahoo.com
}

MS received 3 December 2017; revised 19 March 2018; accepted 2 April 2018; published online 20 July 2018

\begin{abstract}
This study presents numerical and experimental investigation on breast cancer tumour parameters by inverse heat transfer method using genetic algorithm (GA) and image processing (IP) to determine the depth and rate of heat generation of a breast cancer tumour. To simulate the problem, using the energy equation in a cylinder including a heater, the surface temperature distribution was obtained. Then, the temperature surface of the cylinder was analysed by the GA in MATLAB software to determine the depth and rate of heat generation of heater. The validity of the numerical method was evaluated using the IP from a laboratory sample. A thermal heater was placed inside a cylinder and was covered by a tissue similar to the human body tissue. According to the obtained results, it was determined that the results of the laboratory sample and the numerical method were in agreement with each other. Finally, these steps were applied on the thermal image of a patient's cancer breast to determine the depth and rate of heat generation of the breast tumour. It is shown that the average computational error between numerical and experimental results in this method to determine the depth of the tumour is about $8-10 \%$ and to determine the rate of tumour heat generation is about $0.01-1 \%$.
\end{abstract}

Keywords. Thermal images; depth; rate of heat generation; breast cancer tumour; genetic algorithm.

\section{Introduction}

From years ago, body temperature has been considered as one of the important diagnostic indicators in determining the status of human health. It is obvious that with abnormal cell metabolism and disturbance, its function, for any reason, including cancer, causes abnormal changes in body temperature. Temperature on the surface of the body (skin) varies from a maximum of $34.5^{\circ} \mathrm{C}$ on the forehead to a minimum of $27.1^{\circ} \mathrm{C}$ at the fingers. In most cases, the asymmetry of the body temperature map is a reflection of an abnormal activity at lower levels of the skin.

Thermography is a completely safe technique for providing a temperature map from different surfaces of the body. In this method, the patient is placed in front of a thermal camera and the infrared waves emitted from his body are recorded and processed by the camera [1]. Thermography is currently one of the most commonly used complementary techniques for diagnosis of benign and malignant breast diseases. Another method is mammography but the major problem with this method is the

*For correspondence absorption of harmful radiation by the breast tissue, which is not recommended for women under the age of 30 and pregnant women. Nowadays, according to the studies, it has been determined that thermographic imaging has a precision of $95 \%$ for the diagnosis of a person's health or illness, taking into account the correct diagnostic responses against the limited number of positive or negative false responses received; however, it is not a completely precise and absolute method, but complementary, along with other diagnostic methods. According to studies conducted in the mid-1950s, it was found that most breast cancers can be detected by increasing the temperature in the cancerous area. The success rate of thermography in breast cancer diagnosis has been estimated to be about $70 \%$ in one of the treatment centres in the United States [2].

Sheikh Hosseini et al [3] introduced a CAD system for identifying and detecting micro-calcification clusters in mammographic images. The proposed algorithm consists of three basic steps. In the first stage, the wavelet transform is applied to mammograms, and the wavelet coefficient is extracted, along with two statistical characteristics as distinctive features of pixels from the point of view of belonging to a micro-calcification seed. Then, using a 
neural network, the initial classification of pixels is performed. In the third step of the algorithm; after removing the resulting pixels from the first step, the remaining objects are examined in terms of the correspondence to a microcalcification of one seed. Ng and Sudharsan [4] developed a superior three-dimensional direct numerical model of a female breast with tumour and for the inverse problem they used stochastic methods. Mital et al [5] used evolutionary algorithms in artificial neural networks (ANN) and genetic algorithms (GAs) to estimate the breast tumour simulation parameters. Saheb Basha and Satya [6] used diagnostic tools for detecting breast cancer using morphological operators and fuzzy clustering based on image processing (IP). They tried to get the best answer using the fuzzy clustering method and the operators in IP on mammograms for separating the cancerous mass. Yang and Lee [7] developed a novel data mining algorithm and parametric analysis protocol for generating knowledge-based diagnostic rules for infrared thermographs. Alipour and Haddadnia [8] proposed a system for intelligent Fine Needle Aspiration (FNA) technique that achieved a precision of $100 \%$ accuracy using 28 features in the form of 5 SVM models. The steps to create an intelligent system for detecting breast cancer are recording microscopic images of an FNA sample, extracting the numerical properties of these images, selecting the separation features, and designing and testing the appropriate classifier. Thamarai and Ireaneus [9] presented a method akin to that of reference [8] to isolate cancer tumours during several stages of separation. Chang and Liou [10] presented a method using neural networks for the diagnosis of breast cancer, which analysed and surveyed these images using the statistical characteristics of mammograms, and were able to some extent succeed in detecting the masses. Ghayoumizadeh et al [11] identified breast cancer based on thermal pattern in infrared images. In their work, IP and artificial intelligence techniques and the asymmetric technique were used. In this paper, thermal features in infrared images taken from 180 subjects have been used to diagnose breast cancer. In this study, small size and volume fibroids were also clearly visible. Mitra and Balaji [12] investigated the estimation of the position and size of a spherical tumour in a human breast using the temperatures obtained on the surface of the breast through a breast thermogram in conjunction with ANN. The first part of the work involved the numerical simulation of heat conduction in a cancerous breast by employing the Pennes bio-heat transfer equation using a finite-element-based commercial solver COMSOL. The surface temperatures thus obtained from these simulations were trained against the tumour parameters using ANN. Sajjadi et al [13] reviewed the effectiveness of thermography in disease diagnosis. The result of this study is that although thermography method is a non-invasive and inexpensive method, it cannot be used directly because of the inability to achieve acceptable diagnostic value in comparison with other diagnostic methods. Rastghalam and Pourghasem [14] analysed asymmetry using spectral probabilistic properties in the images of thermography. For this purpose, using co-occurrence matrices and extraction of possible and directional features from the spectrum of images taken from the right and left breasts, and comparing them with each other, it was concluded that the probability of abnormality of the sample increased by reducing the similarity of the two vectors. Das et al [15] made a numerical analysis to ascertain the presence of a tumour and to estimate its size and location in a tissue. Heat transfer in the tissue was modelled using the Pennes bioheat transfer equation, and was solved using a finite-volume method (FVM). Temperature distributions in the tissues were specific to the tumour grades, its locations and sizes, and they were different from those of a normal tissue. Sanaiei et al [16] investigated a new approach for estimating the depth, size and metabolic heat generation rate of a tumour. For this purpose, the surface temperature distribution of a breast thermal image and a dynamic neural network were used. The research consisted of two steps: forward and inverse. For the forward section, a finite-element model was created. The Pennes bio-heat equation was solved to find surface and depth temperature distributions. Hatwar and Herman [17] analysed a 2D computational phantom of axisymmetric tumorous breast with six tissue layers: epidermis, papillary dermis, reticular dermis, fat, gland and muscle layer, and spherical tumour was used to generate surface temperature distribution and thereby estimate tumour characteristics iteratively using an inverse algorithm based on the Levenberg-Marquardt method. They showed that the combination of transient data and steady-state data makes it possible to simultaneously estimate tumour size, location and blood perfusion. Melo et al [18] investigated an inverse analysis by considering the solution of Pennes bio-heat equation in an attempt to set the value of blood perfusion to several cases. They used a GA coupled with a finite-element method (FEM) model that reproduces the heat generation phenomenon caused by the tumour.

In this work, numerical and experimental investigations are carried out on the breast cancer tumour parameters by inverse heat transfer method using GA and IP. In the direct mode, the surface temperature distribution is obtained by FVM and then in the inverse mode, the temperature surface of the cylinder is analysed by the GA to determine the depth and rate of heat generation of tumour. It is clear from the literature review that less effort was made in simulation of tumour breast cancer using IP with this approach. Also the other difference between present work and other references is using thermal IP by MATLAB software for deleting extra noises in the thermograph breast images for increasing accuracy. 


\section{Mathematical formulation and solution procedure}

\subsection{Methods and materials}

IP is a branch of computer science that provides the application of this technology in a variety of industries using the processing of information obtained from images taken by a digital thermal camera and the application of changes on these images. IP consists of two main branches of image and vision improvement of the machine. Since the environment around us is full of electromagnetic waves, and given the fact that the transmission of digital images is based on the transmission of electromagnetic waves, it is obvious that during the transfer of information, as well as during the recording of data, noises are applied to the images, which causes reduced image quality. It is here that the branch of image enhancement in IP science helps the researchers in the field to provide clearer images and better quality to carry out their studies. Image enhancement is usually resulted by applying fading filters, and increasing the image contrast results in increased contrast of the image; however, machine vision addresses the ways that can help them get better understanding of the surrounding environment using the images taken and gives the data to machines and robots.

In this study, all computations and the IP were performed using MATLAB software. In MATLAB software, all computations are based on matrices, meaning that the software considers each input, either a number or image, as a matrix and applies different calculations based on the matrix computation on the input. This feature of MATLAB is a strong point in IP because each image contains a number of pixels in a two-dimensional space, and each matrix contains a number of layers in the same space, that is, in a two-dimensional space.

\subsection{Description of the problem}

The primary objective of this study is investigation of the possibility of using thermal images to detect the depth and rate of heat produced in a cancer tumour, which is evaluated by IP. In order to simulate the problem, a thermal heater with a specific depth and rate of heat generated is considered in a cylinder (figure 1a). The radius and height of the cylinder is considered equal to 0.07 and $0.16 \mathrm{~m}$, respectively. The heater radius is assumed to be $0.023 \mathrm{~m}$ and the region of the heater has different thermal properties than the rest of the domain representing healthy tissue. Clearly, this feature makes it possible to store and restore the properties of each pixel separately in an entry of matrices.

In order to determine the temperature of the cylindrical surface (equivalent to the surface temperature of the body), the governing equation for the energy transfer inside the cylinder is solved by FVM. In the next step, the thermal image prepared from the cylinder surface temperature is analysed using the GA in MATLAB software and the depth and the rate of the heat generated are determined.

In the next step, we tried to evaluate the reliability of breast cancer diagnosis using a laboratory model and the $\mathrm{GA}$, and processing the thermal images taken from the laboratory model. A numerical model was employed with experimental data in order to test the validity of this model. As shown in figure $1 \mathrm{~b}$, the heater is located inside a jellylike material akin to the breast tissue, and provides the desired temperature for the simulation of the cancerous tumour with the aid of a DC power supply. A micrometre is

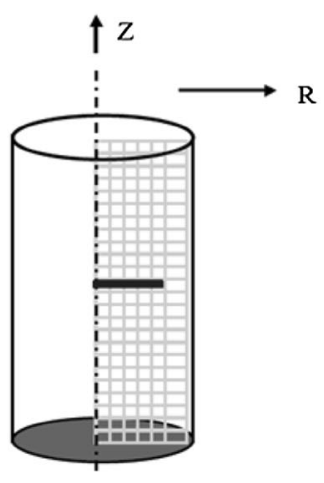

(a)

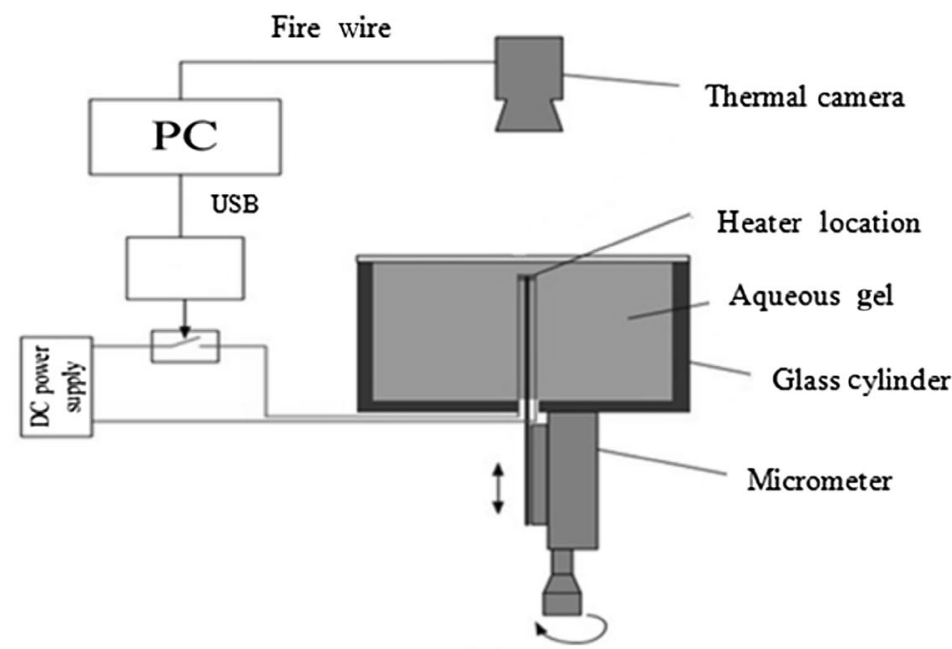

(b)

Figure 1. (a) Schematic of the problem. (b) Set-up for imaging of cylinder surface temperature (heated by a heater). 
also used to change the depth of the heater. The thermal images are recorded using a thermal camera and processed using a computer. The set-up consisted of a lexan circular cylinder filled with agar phantom and mounted on top of a square base plate. Four equally spaced holes were drilled into the cylinder at different depths for inserting the heater wire. The cylinder was covered with 0.02 -m-thick natural cork strip insulation around its circumference. The cylinder was insulated on all sides except the top surface, which was exposed to convection. A resistance heater was embedded in agar and connected to a $0-60 \mathrm{~V} \mathrm{DC}$ power supply. The heater has a radius of $0.023 \mathrm{~m}$ and a resistance of $32 \Omega$. A digital multimeter was set up to probe the voltage across the heater. The FLIR E50 model of the infrared camera from Electrophysics was used for thermal imaging (Appendix). A $1 \%$ solution of Difco Agar was prepared by dissolving the agar in boiling water. The solution was then allowed to cool for a few hours until it acquired semi-solid jelly-like consistency. The thermal and physical properties for the considered gel have been presented in table 1. These steps will ultimately be used for a true image of breast cancer in one patient after the results are verified.

\subsection{Governing equations}

All of the heat transfer processes follow the energy equation; according to the conditions and type of heat transfer, the equation can include conduction or convection and radiation heat transfer. This equation is known as bio-heat equation in biological processes. The two main mechanisms of body heat transfer are conduction heat transfer due to the heat gradient inside the body tissue as well as convection heat transfer from the blood flow to the body tissue (blood perfusion). The bio-heat equation in steady state is expressed as follows:

$$
k \nabla^{2} T+\rho C \omega\left(T_{a}-T\right)+\dot{Q}_{m}=0
$$

where $k$ is the conduction heat transfer coefficient inside the tissue, $T$ is the unknown temperature inside the tissue, $\omega$ is the blood perfusion rate, $C$ is the specific blood heat capacity, $\rho$ is the density of blood and $\dot{Q}_{m}$ is the heat generation rate of the body (metabolic process). It is noticeable that direct oxygen electrode measurements have shown that breast cancer is human tumours reported to have mean oxygen pressures below those of surrounding tissue; despite a wide range of values, the oxygen pressure in tumours tends to decrease with increasing tumour size [19]. Therefore, in the present work the steady state has been considered. In the present work, for simplicity, the entire breast was assumed to be a gland with fixed properties. It is noticeable that most of the breast cancer originates from glandular tissue [19]. The thermal and physical properties for the breast tissue and breast tumour have been presented in table 1 .

Blood perfusion has been considered as a constant parameter in calculations. As already mentioned, in this case, the breast tissue is simulated by a cylinder and the cancerous tumour is simulated using a thermal heater.

In the solution process of bio-heat equation by FVM, the bottom surface is isothermal with arterial blood temperature at $T_{a}=37^{\circ} \mathrm{C}$. The upper (skin) surface is exposed to the environment, exchanging heat with it at $T_{a m b}=21.5^{\circ} \mathrm{C}$, and the convective coefficient is $h=13.5 \mathrm{~W} / \mathrm{m}^{2} \mathrm{~K}$ [18]. The cylinder was considered to be insulated on lateral surface.

\subsection{Solution procedures}

2.4a The direct problem: Since the control volume is intended to be cylindrical, the bio-heat equation must be rewritten in cylindrical coordinates $(r, z)$. The form of this equation in cylindrical coordinates and in a steady state is as follows:

$$
\frac{1}{r} \frac{\partial}{\partial r}\left(k r \frac{\partial T}{\partial r}\right)+\frac{\partial}{\partial z}\left(k \frac{\partial T}{\partial z}\right)+S(r, z, T)=0
$$

where $S(r, z, T)$ is the term associated with the thermal source that can be divided into two parts $S_{C}(r, z)$ and $S_{p}(r, z, T)$. The first part is the conduction heat transfer into the tissue, and the second part is related to the convection heat transfer of a blood perfusion. As a result, this equation can be rewritten as follows:

$$
\frac{1}{r} \frac{\partial}{\partial r}\left(k r \frac{\partial T}{\partial r}\right)+\frac{\partial}{\partial z}\left(k \frac{\partial T}{\partial z}\right)+S_{C}(r, z)-S_{p}(r, z, T) T=0 .
$$

\begin{tabular}{|c|c|c|c|c|}
\hline Parameter & Symbol & Tissue & Tumour & Agar gel \\
\hline Thermal conductivity (W/m K) & $k$ & 0.48 & 0.48 & 0.7 \\
\hline Metabolic heat generation rate $\left(\mathrm{W} / \mathrm{m}^{3}\right)$ & $\dot{Q}_{m}$ & 550 & 32000 & - \\
\hline Density of blood $\left(\mathrm{kg} / \mathrm{m}^{3}\right)$ & $\rho$ & 1050 & 1050 & 1000 \\
\hline Perfusion rate of blood $(\mathrm{ml} / \mathrm{s} / \mathrm{ml})$ & $\omega$ & 0.00018 & 0.009 & - \\
\hline Arterial blood temperature $(\mathrm{K})$ & $T_{a}$ & 310 & 310 & - \\
\hline Specific heat of blood (J/kg K) & $C$ & 3800 & 3800 & 4200 \\
\hline
\end{tabular}

Table 1. The thermal and physical properties for the breast tissue, breast tumour and the considered gel [14]. 


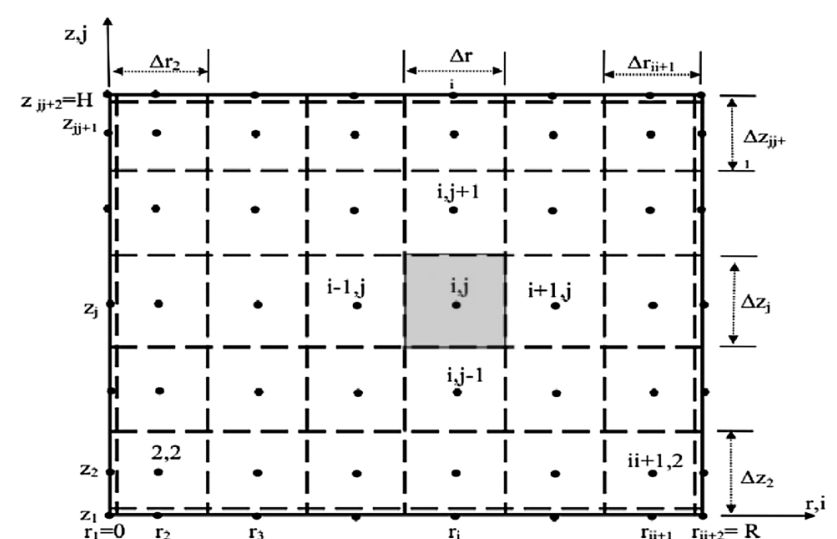

Figure 2. Division of control volume in radial and axial directions.

Since this equation is used on all internal cells of control volume, with an integration on the volume, we will have

$$
\begin{aligned}
& \iint_{S W}^{N E}\left[\frac{1}{r} \frac{\partial}{\partial r}\left(k r \frac{\partial T}{\partial r}\right)+\frac{\partial}{\partial z}\left(k \frac{\partial T}{\partial z}\right)\right. \\
& \left.+S_{C}(r, z)-S_{p}(r, z, T) T\right] \cdot r \cdot d r \cdot d z=0 .
\end{aligned}
$$

Since the aforementioned equation is solved by an FVM, it is necessary to divide the control volume into smaller portions (discretized form) in order to obtain the temperature distribution. Using the boundary conditions for neighbour cells located around the central cell, the direct solution is completed (figure 2). As shown in figure 3, the central cell temperature directly depends on the temperature of the neighbour cells, which include the northern cell $(\mathrm{N})$, the southern cell $(\mathrm{S})$, the eastern cell $(\mathrm{E})$ and the western cell (W). The northern and southern cells are

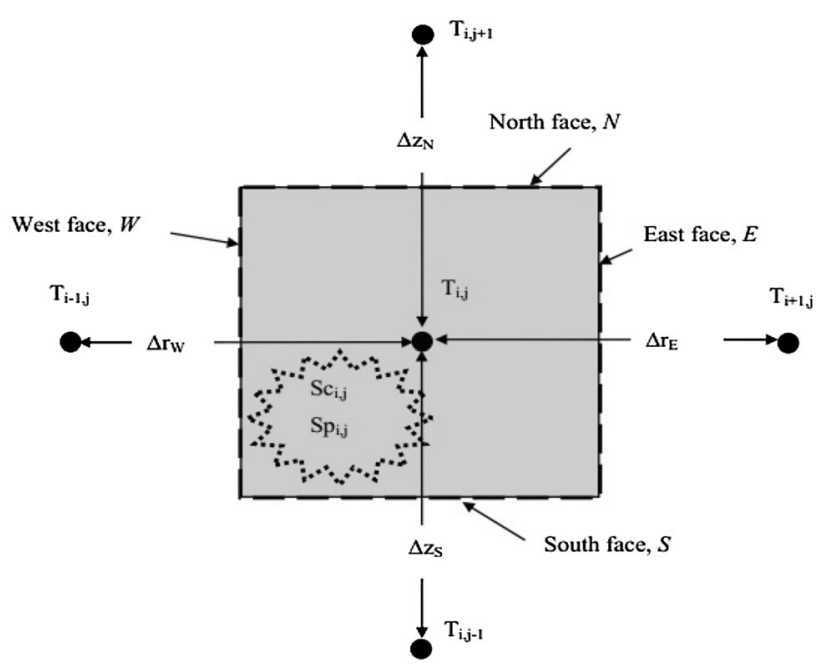

Figure 3. A view of the central cell and the neighbour cells. located in a cylindrical coordinate along the $Z$ and the eastern and western cells along the radius $(r)$.

2.4b The inverse problem: The goal of the problem solution by inverse method is to retrieve the height and heat generation rate of the tumour from the temperatures calculated or measured on the surface of the breast. In this work a GA is used as the parameter estimation method. The GA is based on the evolution of generations and a learning method based on biological evolution, introduced in 1970 by Johan Holland. The GA generates a large set of possible solutions to solve a problem and then evaluates these solutions with the help of the fitness function and selects some of the best solutions. In fact, the GA seeks to find the best answer among the innumerable hypotheses (population). At each stage, a set of hypotheses is obtained by replacing a part of the current population with the children who have obtained the best of the available answers. The concept of GAs has been modified for use with the problem of detecting embedded tumours. The potential solution (chromosome) contains the tumour height and its heat generation rate as the two genes.

The objective function used here is a sum of the squares function that relates the data from the model to the corresponding experimental data. It measures the distance between the estimated and the calculated or measured surface temperature distributions. The sum of the squares is defined as follows:

$$
S=[Y-T(\beta)]^{T}[Y-T(\beta)]
$$

where $\mathbf{Y}$ and $\mathbf{T}$ are vectors containing the calculated or measured and estimated temperatures, respectively, and superscript $\mathrm{T}$ denotes the transpose of the vector. The estimated temperatures are obtained by the solution of the direct problem using estimates for the unknown parameters. The vector containing the estimates for unknown parameters is denoted by $\beta$. In this study, the unknown parameter vector $\beta$ contains the depth and the heat generation rate of the tumour. The population size has been chosen to be 20 . The fitness of each chromosome is evaluated using the sum of the squares objective function given by Eq. (5). The fitness $f$ of a chromosome is related to the sum of the squares function using

$$
f=S_{\text {MAX }}-S(\beta)
$$

where $S_{M A X}$ is the maximum value of sum of squares possible and $S$ is the sum of squares generated using parameter set (chromosome) $\beta$.

The finite-volume code takes the height and heat generation information from an individual chromosome and determines the temperature response of the tissue. The closer the finite-volume temperature response is to the actual temperature response, the lower the sum of squares $S$, and the higher the fitness of that chromosome. Chromosomes of higher fitness are more likely to be selected to reproduce and contribute their genetic material to the next 
generation. A probability of mutation of 0.08 and a probability of crossover equal to 0.35 are used to determine how members of the population will reproduce to yield for the next generation. A flowchart of the solution method has been presented in figure 4 .

\section{Discussion and conclusions}

\subsection{Numerical validation}

For the validity of solution, after analysing the governing equations and applying the boundary conditions in MATLAB code, the results were obtained regarding the depth and heat generation rate. Therefore, before performing the test on the thermal image sample, it is required to evaluate the program using the predetermined depth and heat generation rate in order to determine the error rate of the algorithm used. In this regard, the depth of the heater and heat generation rate are considered as predetermined values. After the implementation of the program, the results of table 2 are obtained:

In table 2, the values of the depth and heat generation rate are specified in numerical mode. First, by entering actual values (depth and heat generation rate) in MATLAB software, the cylindrical surface temperature distribution is obtained. In the next step, the obtained surface temperature is entered into the GA and by comparing the results obtained at each time the GA is executed with the surface temperature vector of the previous stage. In this way, the closest and most optimal solution for the depth and heat generation rate is obtained. As is obvious, the program has been run twice to ensure the correct execution of the computational code written for the same depth and heat generation rate. For example, for the repetition of $\mathrm{A} 1$ and A2, a depth of $4.12 \mathrm{~cm}$ and a heat generation rate of $0.015 \mathrm{~W} / \mathrm{cm}^{2}$ were considered. After executing the code of the GA, for the first time, the depth of $3.4839 \mathrm{~cm}$ and the heat generation rate of $0.0161 \mathrm{~W} / \mathrm{cm}^{2}$ were considered, and for the second time these values were calculated as $3.67 \mathrm{~cm}$ and $0.0161 \mathrm{~W} / \mathrm{cm}^{2}$, respectively. On comparing the obtained values and comparing their changes with the exact values, the efficiency of the GA can be trusted in solving this problem.

The results obtained in figures 5 and 6 are for the sole purpose of showing the validity of the GA, and these results are obtained from the predetermined values.

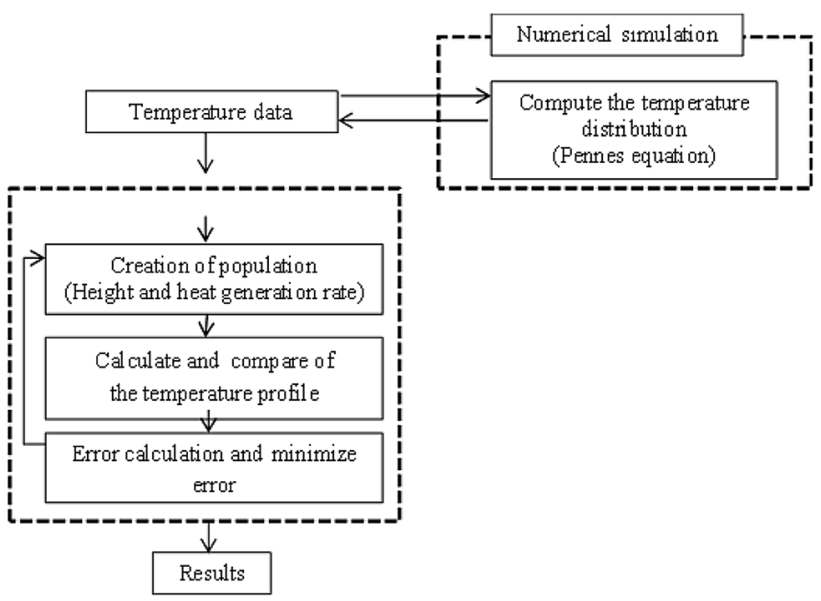

Figure 4. GA-based estimation procedure coupled with FVM.

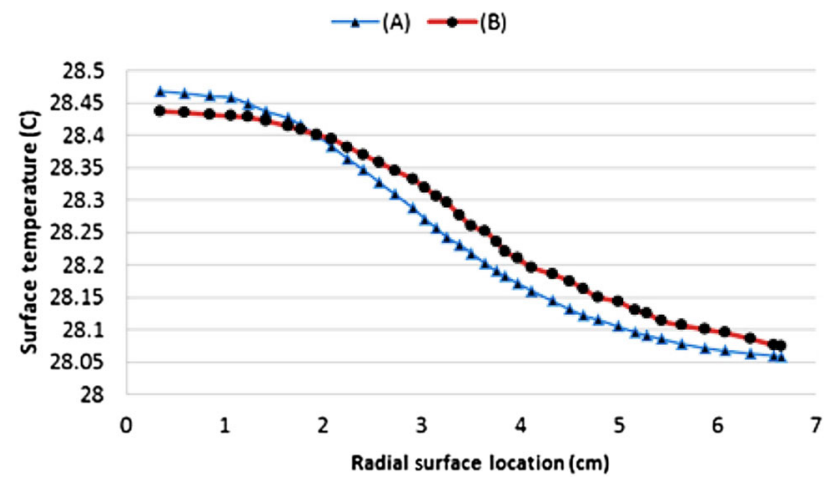

Figure 5. Variation in cylinder surface temperature with respect to the depth and rate of heat generation based on case A1: (A) height $=4.12 \mathrm{~cm}$ and heat generation rate $=0.015 \mathrm{~W} / \mathrm{cm}^{2}$ and (B) height $=3.4839 \mathrm{~cm}$ and heat generation rate $=0.0161 \mathrm{~W} / \mathrm{cm}^{2}$.

Table 2. Comparison between heater depth and heat generation rate for exact and numerical modelling.

\begin{tabular}{lcccccc}
\hline Iter. & $\begin{array}{c}\text { Exact height } \\
(\mathrm{cm})\end{array}$ & $\begin{array}{c}\text { Exact heat generation } \\
\left(\mathrm{W} / \mathrm{cm}^{2}\right)\end{array}$ & $\begin{array}{c}\text { Height }(\mathrm{cm}) \text { by numerical } \\
\text { method }\end{array}$ & $\begin{array}{c}\text { Heat generation }\left(\mathrm{W} / \mathrm{cm}^{2}\right) \text { by } \\
\text { numerical method }\end{array}$ & $\begin{array}{c}\Delta \dot{Q}_{m}(\mathrm{~W} / \\
\left.\mathrm{cm}^{2}\right)\end{array}$ \\
\hline A1 & 4.12 & 0.015 & 3.4839 & 0.0161 & 0.0161 & 0.0011 \\
A2 & 4.12 & 0.015 & 3.67 & 0.0161 & 0.45 \\
B1 & 2.15 & 0.3 & 2.12 & 0.3065 & 0.001 \\
B2 & 2.15 & 0.3 & 2.129 & 0.3065 & 0.03 \\
C1 & 3.5 & 0.5 & 3.4839 & 0.5 & 0.0065 \\
C2 & 3.5 & 0.5 & 3.49 & 0.5 & 0.006 \\
\hline
\end{tabular}




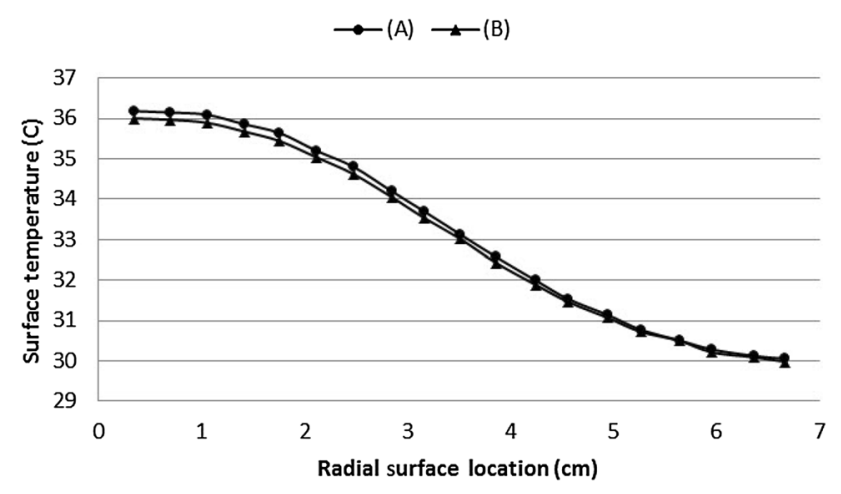

Figure 6. Variation in cylinder surface temperature with respect to the depth and rate of heat generation based on case B1: (A) height $=2.15 \mathrm{~cm}$ and heat generation rate $=0.3 \mathrm{~W} / \mathrm{cm}^{2}$ and (B) height $=2.12 \mathrm{~cm}$ and heat generation rate $=0.3065 \mathrm{~W} / \mathrm{cm}^{2}$.

Figure 5 shows the changes in cylinder surface temperature with respect to the depth and rate of heat generation in exact case with height equal to $4.12 \mathrm{~cm}$ and heat generation rate equal to $0.015 \mathrm{~W} / \mathrm{cm}^{2}$ and predicted by $\mathrm{GA}$ with height equal to $3.4839 \mathrm{~cm}$ and heat generation rate equal to $0.0161 \mathrm{~W} / \mathrm{cm}^{2}$ (case A1). It can be observed that the surface temperature values have similar temperature profile. A lower value of radial surface location causes the temperature profile to move up, while a higher value makes it move down. The changes in cylinder surface temperature with respect to the depth and rate of heat generation of the heater for another case B1 have been shown in figure 6. It can be observed that with increasing of radial surface location the surface temperature decreased.

Figure 7 shows the surface temperature for three different heater radii. As is shown in this diagram, increasing the radius of the heater reduces the maximum cylindrical surface temperature, but the area that has been exposed to the

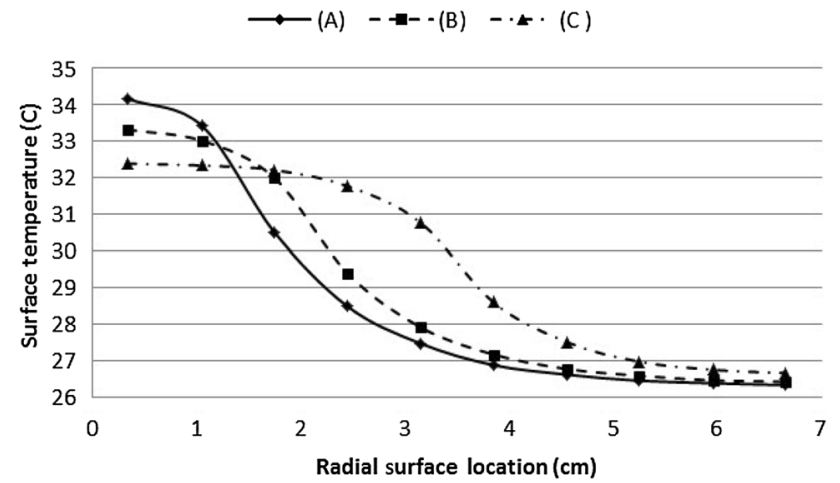

Figure 7. Changes in cylinder surface temperature with respect to the depth, radius and the rate of heat generation: (A) radius $=0.14$ $\mathrm{cm}$, height $=5.6129 \mathrm{~cm}$ and heat generation rate $=0.2097 \mathrm{~W} / \mathrm{cm}^{2}$, (B) radius $=0.28 \mathrm{~cm}$, height $=5.6129 \mathrm{~cm}$ and heat generation rate $=$ $0.1129 \mathrm{~W} / \mathrm{cm}^{2}$ and $(\mathrm{C})$ radius $=0.42 \mathrm{~cm}$, height $=5.6129 \mathrm{~cm}$ and heat generation rate $=0.645 \mathrm{~W} / \mathrm{cm}^{2}$. maximum temperature has expanded. We will see in detail in the three diagrams that in fact the shape of the three graphs is almost the same and the only difference lies in the thermal range of the heater.

In the range of the heater performance, because the surface temperature of the heater is uniform, the cylinder surface temperature remains the same, which is more pronounced in the case of a radius of $0.28 \mathrm{~cm}$, but outside the heater performance. The behaviours of all three graphs are completely identical. As is evident, the behaviour of the diagram outside the range of the heater is exactly the same, which indicates that the radius of the heater has no effect on the problem-solving process, and the results of all three steps can be considered as the answer to the problem.

Figure 8 shows the changes resulting from the change in the conduction heat transfer coefficient inside the cylinder (tissue). As is clear, with the increase in the conduction heat transfer coefficient, the cylindrical surface temperature at the centre near the heater is reduced. However, as the diagram shows, the changes in the surface temperature of the cylinder are not appreciable; in fact, the changes in the conduction heat transfer coefficient do not have a significant effect on the problem-solving process.

\subsection{Experimental results}

For determination of the depth and heat generation rate of tumour by IP using GA, the accuracy and performance of this method must be evaluated. The images obtained from the experimental set-up (cylinder and heater) are for confidence of numerical calculations. After confirmation from correctness of results for the sample, the images of the cancerous breast of the patient are investigated. In figure 9, the recorded thermal image of the surface of the cylinder heated by the internal heater (equivalent to the tumour), located at a certain depth of the cylinder surface (equivalent to the surface of the skin) and emitting a certain amount of

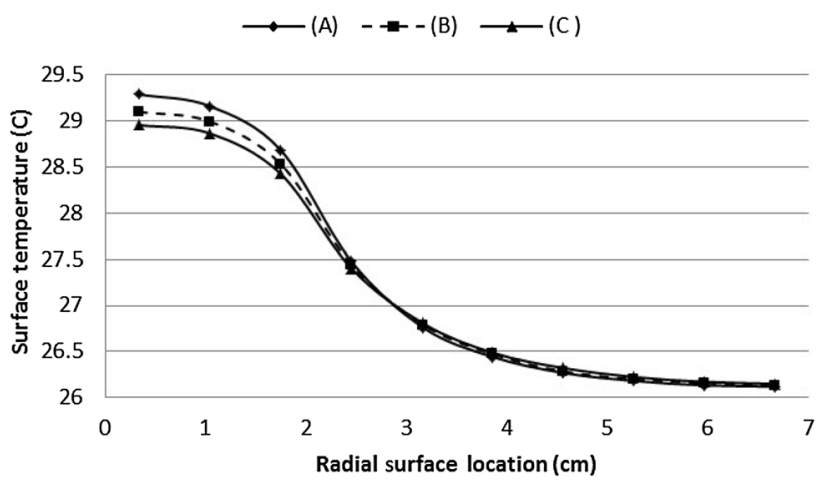

Figure 8. Indication of changes in cylinder surface temperature due to changes in the conduction heat transfer coefficient $(k)$ for height $=6 \mathrm{~cm}$ and heat generation rate $=0.484 \mathrm{~W} / \mathrm{cm}^{2}$ : (A) $k=$ $3.8 \mathrm{~W} / \mathrm{m}{ }^{\circ} \mathrm{C}$, (B) $\mathrm{k}=4.8 \mathrm{~W} / \mathrm{m}^{\circ} \mathrm{C}$ and $(\mathrm{C}) k=5.8 \mathrm{~W} / \mathrm{m}{ }^{\circ} \mathrm{C}$. 


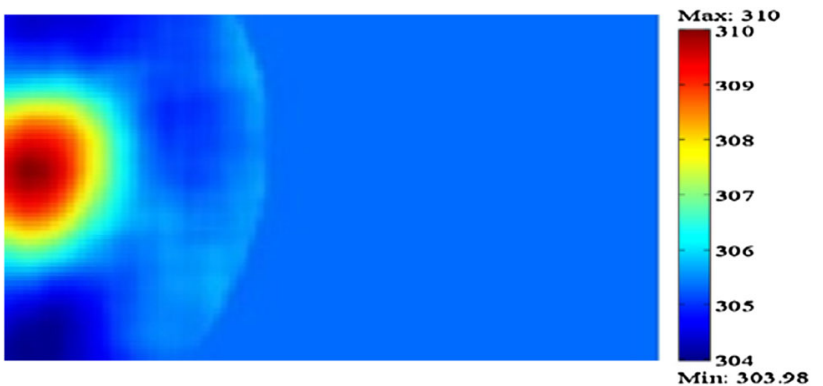

Figure 9. The thermal image taken from the cylinder surface due to the heating of internal heater (surface temperature distribution).

heat (identifying the type of tumour) has been shown. This image is inserted into the GA after the IP by MATLAB software and excessive noise is removed in the form of a two-dimensional matrix in which the colours determine the colour of each pixel. Finally, the depth and the rate of heat generation of the heater are determined in a reverse process.

Table 3 shows the surface temperature comparison between results obtained from infrared camera and predicted values by GA with height equal to $3 \mathrm{~cm}$ and heat generation rate $0.03 \mathrm{~W} / \mathrm{cm}^{2}$ for different radii of the heater. It can be observed that the heater with $r=2.5 \mathrm{~cm}$ has similar values with data obtained from the infrared camera.

In this study, the thermal image of a breast cancer patient was examined and the results were evaluated and compared. Figure 10 shows thermograph image of a patient with breast cancer at right hand side and healthy breast at left hand side. The test environment plays an important role in determining the boundary conditions.

In figure 11, the results of IP for image 10 have been shown for three different radii of the tumour. As is obvious, although the value of the considered radius for the tumour increases, the calculated depth changes are not tangible and only the heat generation rate changes. It is noted that with increasing radius for the tumour, the temperature of the heat-emitting tumour and also the maximum surface temperature of the skin increases.

In figure 12, the results of IP in figure 10 are shown for the second repetition and three different tumour sizes. As

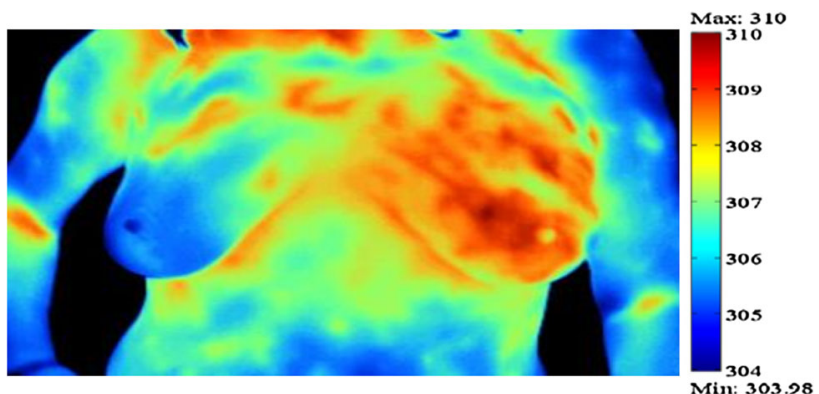

Figure 10. Thermal image taken from a patient with breast cancer.

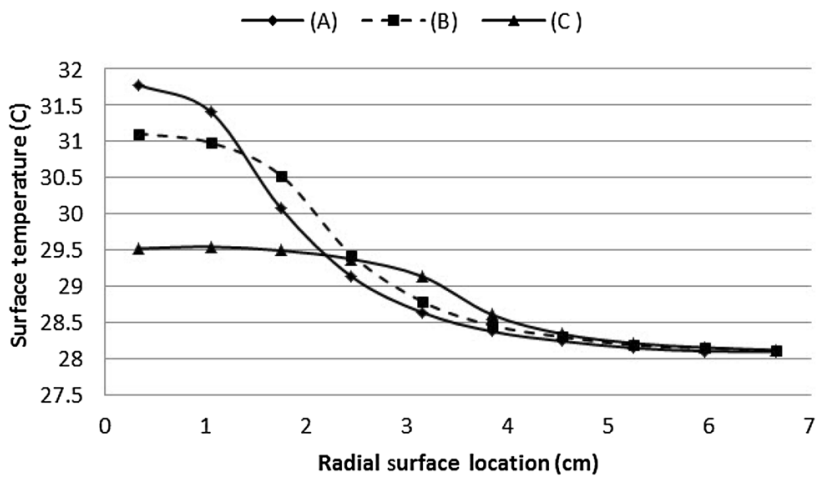

Figure 11. Variation in body surface temperature with respect to the depth, radius and rate of heat generation of the tumour: (A) radius $=0.42 \mathrm{~cm}$, height $=5.6134 \mathrm{~cm}$ and heat generation rate $=0.968 \mathrm{~W} / \mathrm{cm}^{2},(\mathrm{~B})$ radius $=0.28 \mathrm{~cm}$, height $=5.3832 \mathrm{~cm}$ and heat generation rate $=0.484 \mathrm{~W} / \mathrm{cm}^{2}$ and $(\mathrm{C})$ radius $=0.14 \mathrm{~cm}$, height $=$ $5.2123 \mathrm{~cm}$ and heat generation rate $=0.161 \mathrm{~W} / \mathrm{cm}^{2}$.

expected, the results are subject to slight variations compared with the previous one.

Finally, IP carried out for the healthy breast of figure 10 and then for different radii codes run and the depth and heat generation rate were determined. Figure 13 shows the thermal image analysis taken from the healthy breast. As the figure shows, the maximum temperature change at the

Table 3. Surface temperature $\left({ }^{\circ} \mathrm{C}\right)$ at different radial surface locations for different radii of the heater.

\begin{tabular}{lcccccc}
\hline Nodal surface temperatures from infrared camera & $\mathrm{r}=0.5 \mathrm{~cm}$ & $\mathrm{r}=1.0 \mathrm{~cm}$ & $\mathrm{r}=2.0 \mathrm{~cm}$ & $\mathrm{r}=2.5 \mathrm{~cm}$ & $\mathrm{r}=4.0 \mathrm{~cm}$ & $\mathrm{r}=6.0 \mathrm{~cm}$ \\
\hline 33.01 & 32.97 & 32.98 & 33.17 & 33.03 & 31.05 \\
32.95 & 32.9 & 32.91 & 33.13 & 32.98 & 31.04 & 31.62 \\
32.92 & 32.64 & 32.63 & 32.97 & 32.86 & 31.01 \\
32.51 & 32.1 & 32.06 & 32.63 & 32.56 & 30.95 \\
31.95 & 31.32 & 31.24 & 32.07 & 32.04 & 30.83 \\
31.32 & 30.42 & 30.31 & 31.33 & 31.25 & 30.62 \\
30.36 & 29.54 & 29.42 & 30.51 & 30.28 & 30.23 \\
29.27 & 28.8 & 28.67 & 29.75 & 29.37 & 29.49 \\
28.69 & 28.27 & 28.15 & 29.16 & 28.73 & 28.01 \\
28.3 & 27.99 & 27.87 & 28.85 & 28.34 & 27.4 & 31.42 \\
\hline
\end{tabular}




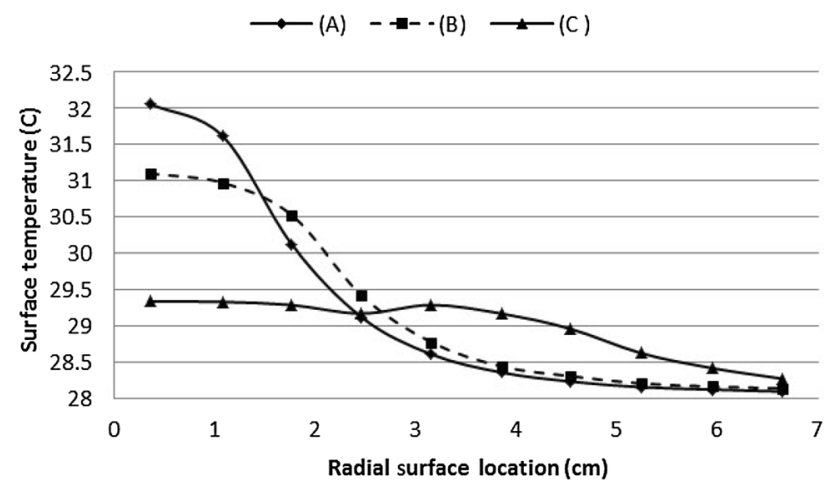

Figure 12. Variation in surface temperature in terms of the depth, radius and the rate of heat generation of the tumour. (A) Radius $=0.42 \mathrm{~cm}$, Height $=5.8065 \mathrm{~cm}$ and Heat Generation Rate $=0.968 \mathrm{~W} / \mathrm{cm}^{2},(\mathrm{~B})$ radius $=0.28 \mathrm{~cm}$, height $=5.6129 \mathrm{~cm}$ and heat generation rate $=0.484 \mathrm{~W} / \mathrm{cm}^{2}$ ) and $(C)$ radius $=0.14 \mathrm{~cm}$, height $=5.2258 \mathrm{~cm}$ and heat generation rate $=0.161 \mathrm{~W} / \mathrm{cm}^{2}$.

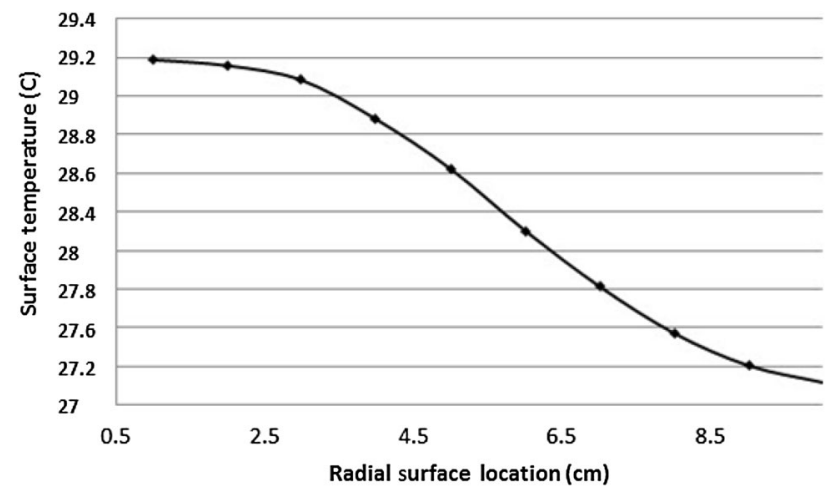

Figure 13. Changes in body surface temperature in healthy breast. radius $=0.14 \mathrm{~cm}$, height $=0.13 \mathrm{~cm}$ and heat generation rate $=0.09 \mathrm{~W} / \mathrm{cm}^{2}$.

centre of the tumour is equal to normal temperature of body. On the other hand, the variation of surface temperature is negligible because the depth of the tumour is very high (low height) and its rate of heat generation is very low. Considering this, the presence of a cancerous tumour in this area is excluded from the body.

\section{Conclusion}

This study presents the numerical and experimental investigation on the breast cancer tumour parameters by inverse heat transfer method using GA and IP to determine the depth and rate of heat generation of a breast cancer tumour. To simulate the problem, using the energy equation in a cylinder including a heater, the surface temperature distribution was obtained. Then, the temperature surface of the cylinder was analysed by the GA in MATLAB software to determine the depth and rate of heat generation of heater. The validity of the numerical method was evaluated using the IP from a laboratory sample. Finally, these steps were applied on the thermal image of a patient's cancer breast to determine the depth and rate of heat generation of the breast tumour. The following results were obtained from this analysis:

1. Based on the obtained diagrams, it was determined that the results of the simulated model and the real sample were satisfactorily matched with each other and, in the same conditions, the behaviours of the graph from the laboratory sample and the simulated sample were the same and that minor variations in the diagrams were simply related to the conditions and the way of doing thermal imaging.

2. The subject that may at first glance be of particular importance in determining the depth of the tumour is the size of the radius or the extent of the spread of the tumour, which can be effective in determining the depth and rate of heat generation of the tumour. In order to investigate this, in different modes and using heaters with different radii, the experiment was repeated, with preliminary results suggesting a decrease in the maximum surface temperature and also a decrease in the heat output of the heater. This confirms the direct effect of the tumour radius on the predictive accuracy of this diagnostic method; however, further studies show that the performance of all three charts is the same outside the tumour area and the changes in the graphs are related to the region directly affected by the temperature of the heater and therefore outside the tumour. The behaviours of the graphs are completely identical; thus, in any case, for any size and radius of the tumour, the method is capable of calculating and determining the depth and rate of heat generation without any impairment. On the other hand, the reason for the decrease of the maximum surface temperature and the rate of heat production is the distribution of the voltage applied to the heater at a wider radius of the environment, and this has nothing to do with the radius of the tumour under the same conditions in the real sample. It was also found that this method, in the case of smaller size and radius of tumour, has the ability to detect mass more than that in the case where tumour has been expanded. However, in the case of cancerous tumour spreading, it is possible to consider the tumour radius as small as possible to the desired result on the depth and rate of heat generation of the tumour.

3 . The average computational error in this method to determine the depth of the tumour was calculated to be $8-10 \%$ and the rate of tumour heat generation was $0.01-1 \%$.

\section{Nomenclatures}

C specific heat capacity, $\mathrm{J} / \mathrm{kg} \mathrm{K}$

$h$ average convection coefficient, $\mathrm{W} / \mathrm{m}^{2} \mathrm{~K}$

$k$ thermal conductivity, W/m K

$\dot{Q}_{m}$ heat generation, $\mathrm{W} / \mathrm{m}^{3}$ 
$r \quad r$-coordinate

$R$ radius of the tumour, $\mathrm{m}$

$S \quad$ sum of squares

$T$ temperature, ${ }^{\circ} \mathrm{C}$

$t$ time, s

Y calculated or measured temperature vector

\section{Greek symbols}

$\beta$ unknown parameter vector

$\rho$ density

$\omega$ blood perfusion term

\section{Subscripts \\ a arterial \\ $b$ blood \\ $m$ metabolic}

\section{Superscripts}

$\mathrm{T}$ transpose

\section{Appendix}

Specification for the FLIR E50 infrared camera.

\begin{tabular}{|c|c|}
\hline \multicolumn{2}{|l|}{ Imaging performance } \\
\hline IR resolution & $240 \times 180$ pixels \\
\hline Spatial resolution & $1.82 \mathrm{mrad}$ \\
\hline Thermal & $<0.05^{\circ} \mathrm{C}$ \\
\hline Sensitivity zoom & $\begin{array}{l}1-4 \times \text { continuous digital zoom, incl } \\
\text { panning }\end{array}$ \\
\hline \multicolumn{2}{|l|}{ Image presentation } \\
\hline Picture in picture & Scalable IR area on visual image \\
\hline Thermal fusion & Yes \\
\hline Image modes & $\begin{array}{l}\text { IR image, visual image, thermal } \\
\text { fusion, picture-in-picture, } \\
\text { thumbnail gallery }\end{array}$ \\
\hline \multicolumn{2}{|l|}{ Measurement } \\
\hline $\begin{array}{l}\text { Object temperature } \\
\text { range }\end{array}$ & -20 to $+120^{\circ} \mathrm{C} / 0$ to $+650^{\circ} \mathrm{C}$ \\
\hline \multicolumn{2}{|l|}{ Measurement analysis } \\
\hline Spot meter & 3 \\
\hline Area & 3 boxes with $\min . / \max . / a v e r a g e$ \\
\hline $\begin{array}{l}\text { Difference } \\
\text { temperature }\end{array}$ & $\begin{array}{c}\text { Delta temperature between } \\
\text { measurement functions or } \\
\text { reference temperature }\end{array}$ \\
\hline $\begin{array}{l}\text { Reporting instant } \\
\text { report }\end{array}$ & N/A \\
\hline $\begin{array}{l}\text { Digital camera built- } \\
\text { in digital camera }\end{array}$ & 3.1 Mpixels, and one LED light \\
\hline \multicolumn{2}{|l|}{ Image annotations } \\
\hline Voice & $60 \mathrm{~s}$ via Bluetooth $\AA$ \\
\hline Text & $\begin{array}{l}\text { Text from predefined list or soft } \\
\text { keyboard on touch screen }\end{array}$ \\
\hline MeterLink & $\begin{array}{l}\text { Bluetooth, Extech moisture meter } \\
\text { MO297 or Extech clamp meter } \\
\text { EX845 }\end{array}$ \\
\hline
\end{tabular}

\section{References}

[1] Tortora G J 2003 Principles of anatomy and physiology. New York: John Wiley \& Sons, Inc.

[2] Jemal J A 2016 Cancer statistics, breast cancer wars. J. Oncol. 6: 147-150

[3] Sheikh H A, Abrishami M H, Giti M, Abdolmalaki P and Mostafavi A 2003 Automatic detection of micro-calcification cluster by wavelet conversion and neural networks. In: Proceedings of the Second Image Processing Conference

[4] Ng E Y K and Sudharsan N M 2006 An improved three-dimensional direct numerical modelling and thermal analysis of a female breast with tumor. Proc. Inst. Mech. Eng. [H] 215: 25-37

[5] Mital M, Ramana M and Pidaparti S 2008 Breast tumor simulation and parameters estimation using evolutionary algorithms. Model. Simul. Eng. 8: 35-46

[6] Saheb Basha S and Satya P 2009 Automatic detection of breast cancer mass in mammograms using morphological operators and fuzzy C-means. J. Theor. Appl. Inf. Technol. 2: 114-120

[7] Yang C S and Lee M Y 2008 Parametric data mining and diagnostic rules for digital thermographs in breast cancer. In: Proceedings of the 30th Annual International Conference of the IEEE Engineering in Medicine and Biology Society, pp. 98-101

[8] Alipour A and Haddadnia J 2009 Introduction of an intelligent system for the diagnosis of breast cancer. In: Proceedings of the Fifth Image Processing Conference

[9] Thamarai S and Ireaneus A 2009 Early detection of breast cancer using SVM classifier technique. Comput. Sci. Eng. 1: 127-133

[10] Chang P W and Liou M D 2010 Comparison of three data mining techniques with genetic algorithm in analysis of breast cancer data. J. Theor. Appl. Inf. Technol. 2: 25-29

[11] Ghayoumizadeh H, Abbaspour Kazerouni I, Haddadnia J and Hashemian M 2011 Identification of breast cancer based on the thermal pattern in infrared pictures. J. Breast Dis. 1: 75-81

[12] Mitra S and Balaji C 2010 A neural network based estimation of tumor parameters from a breast thermogram. Int. J. Heat Mass Transf. 53: 4714-4727

[13] Sajjadi H, Seyyedin S, Zaboli R and Gholami S 2011 The effectiveness of contact thermography in the diagnosis of disease: a systematic review. Razi J. Med. Sci. 18: 11-19

[14] Rastghalam R and Pourghasem H 2013 Breast cancer detection based on asymmetric analysis using spectral probabilistic properties in thermographic images. J. Breast Dis. 2: 135-141

[15] Das K, Singh R and Mishra S C 2013 Numerical analysis for determination of the presence of a tumor and estimation of its size and location in a tissue. J. Therm. Biol. 38: 32-40

[16] Sanaiei E, Setayeshi S, Akbari M E and Navid M 2016 Parameter estimation of breast tumor using dynamic neural network from thermal pattern. J. Adv. Res. 7: 1045-1055

[17] Hatwar R and Herman C 2017 Inverse method for quantitative characterisation of breast tumors from surface temperature data. Int. J. Hyperth. 33: 741-757

[18] Melo A R, Loureiro M M S and Loureiro F 2017 Blood perfusion parameter estimation in tumors by means of a Genetic Algorithm. In: Proceedings of the International Conference of Computational Science, Zurich, Switzerland

[19] Perez C A and Bradys L W 2016 Principle and practice of radiation and oncology, 6th edn, Lippincott Williams Wilkins, USA 